\title{
Acotovelamento de enxerto de artéria torácica interna na revascularização do miocárdio: relato de caso
}

Artur LOURENÇÃO Jr. *, José Carlos R. IGLÉZIAS*, Luís Alberto DALLAN*, Ana Nery R. PEREIRA*, Sérgio Almeida de OLIVEIRA*, Adib D. JATENE*

RBCCV 44205-221

LOURENÇĀO Jr., A.; IGLÉZIAS, J. C. R.; DALLAN, L. A.; PEREIRA, A. N. R.; OLIVEIRA, S. A.; JATENE, A. D. Rev. Bras. Cir. Cardiovasc., 8 (3): 247-249, 1993.

RESUMO: Os autores apresentam caso de revascularizaçāo do miocárdio, complicado por acotovelamento da artéria torácica interna esquerda, tratado com sucesso.

DESCRITORES: artéria torácica interna, acotovelamento; miocárdio, revascularização, cirurgia.

\section{INTRODUÇÃO}

Desde o início da sua utilização na revascularização direta das artérias coronárias, a artéria torácica interna tem-se mostrado superior aos enxertos venosos, apesar dos melhores resultados; cuidados devem ser tomados para evitar problemas que possam comprometer o seu uso. Uma das complicações é a compressão do pedículo arterial pelo pulmão, durante a inspiração, e a solução para evitar tal ocorrência, é a utilização de pedículo o mais longo possível, com o risco do acotovelamento do enxerto.

Outra manobra é a secção ampla da pleura e abertura de uma "janela" no pericárdio para passagem da artéria, colocando-a em posição favorável, onde, mesmo com a hiperinsuflação pulmonar, não há compressão ou estiramento do enxerto.

Apesar dos cuidados técnicos habituais, defrontamo-nos com casos de obstrução parcial ao fluxo da artéria torácica interna resultante de acotovelamentos do enxerto.

\section{RELATO DO CASO}

OFL, 48 anos de idade, branco, com história de angina progressiva aos esforços desde há 5 meses, o que acabou levando-o a limitação física e incapacidade para o trabalho. Realizada cineangiocoronariografia, que revelou lesão de $90 \%$ na origem da artéria interventricular anterior (IVA), $70 \%$ na origem do primeiro ramo diagonal ( $\mathrm{DI}_{1}$ ), $80 \%$ na artéria circunflexa $(C x)$ e origem do ramo marginal esquerdo (ME); a artéria coronária direita (CD) mostrava várias irregularidades, sendo a maior com obstrução de $50 \%$ a $60 \%$ da luz do vaso.

A cirurgia para revascularização do miocárdio, constitui em anastomose da artéria torácica interna esquerda (ATIE) com a IVA, posicionada através de "janela" aberta longitudinalmente no pericárdio, aproximadamente 2 centímetros anterior ao nervo frênico. A artéria torácica interna direita (ATID) foi anastomosada à $\mathrm{ME}$, utilizando-se o trajeto retroaórtico; foram realizadas pontes de veia safena para $\mathrm{DI}_{1}$ e CD no seu ramo interventricular posterior. 
Terminadas as anastomoses, foi necessário o emprego de balão intra-aórtico (BIA) para retirada da circulação extracorpórea (CEC), devido a baixo débito cardiaco transitório.

A evolução pós-operatória inicial foi boa, com remoção do BIA após 12 horas, dada a estabilidade hemodinâmica. Todavia, no $3^{\circ}$ dia de pós-operatório, voltou a apresentar sinais e sintomas de baixo débito cardíaco.

O exame eletrocardiográfico mostrou isquemia da parede anterior do ventrículo esquerdo; concomitantemente, o exame ecocardiográfico revelou acinesia médio-apical e ântero-septal, com disfunção discreta global de VE e ausência de trombos no seu interior. Indicado novo estudo cineangiocoronariográfico, que mostrou as pontes de safena pérvias, bem como a ATIE apresentava pinçamento no $1 / 3$ distal, antes da anastomose com a IVA (Figura 1); ventrículo esquerdo com hipocontratilidade difusa.

A cineangiocoronariografia sugeria compressão da ATIE na altura em que o enxerto ultrapassava a "janela" aberta no pericárdio.

Com esse diagnóstico, o paciente reoperado. Após a abertura do toráx, constatou-se que a "janela" no pericárdio era ampla e não exercia qualquer pressão sobre a ATIE; como o paciente apresentava o coração verticalizado e um certo grau de dilatação do tronco pulmonar, a ATIE ficou posicionada em parte sobre a transição entre o tronco pulmonar e a via de saída do ventrículo direito. Concomitantemente a cada contração ventricular, evidenciava-se 0 aparecimento de um sulco entre o ventrículo direito e o tronco pulmonar dilatado e o pedículo da ATIE acoplado à região, sofria acotovelamento a cada contração, com conseqüente diminuição do fluxo.

Após o deslocamento lateral do pedículo da ATIE e sua fixação mais à esquerda, desapareceu

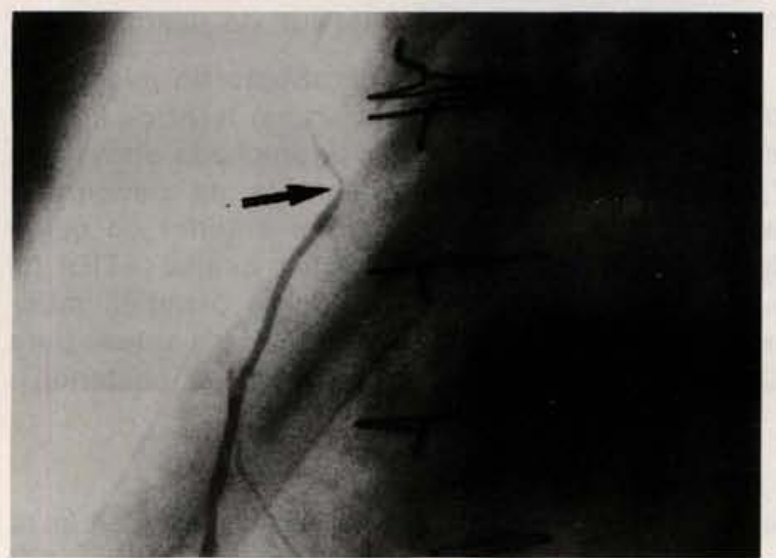

Fig. 1 - Cinecoronariografia em OAE, mostrando o acotovelamento da ATIE (seta); o arco acima é a porção sobre o TP, e abaixo, sobre a via de saída do VD.
- acotovelamento do pedículo, com progressiva melhora da contratilidade ventricular esquerda.

A evolução do paciente transcorreu bem, do ponto de vista hemodinâmico e sem sinais eletrocardiográficos de isquemia.

O paciente recebeu alta no $10^{2}$ dia de pósoperatório, condições clínicas e sem sinais de isquemia miocárdica. $\mathrm{O}$ exame ecocardiográfico após a falta hospitalar, no $20^{2}$ dia, em ambulatório mostrou o VE com função normal, boa fraçào de ejeção e contração segmentar normal.

\section{COMENTÁRIOS}

O emprego de enxertos arteriais na revascularização do miocárdio vem ganhando destaque nos últimos anos. A utilização de ambas as artérias torácicas internas in situ ou como enxerto livre, tem contribuído na melhoria dos resultados cirúrgicos a médio longo prazos, diminuindo a incidência de reoperações 1,2 .

A ATID tem sido utilizada com maior freqüência para os ramos da artéria circunflexa ${ }^{4}$. Temos evitado seu emprego in situ para os ramos arteriais da face anterior do coração, pois seu trajeto retrosternal traz grandes dificuldades técnicas em caso de eventual reoperação.

Estas mesmas considerações são válidas em relação à ATIE; seu posicionamento pré-pericárdico dificulta nova toracotomia, expondo o enxerto a possíveis lesões durante a esternotomia ou na dissecção do coração ${ }^{3}$.

Por isso, atualmente, temos preferido a passagem da artéria através de pequena "janela" aberta no pericárdio, anterior ao nervo frênico. Essa manobra encurta $\circ$ trajeto entre a artéria subclávia, origem da artéria torácica interna (ATI) e a artéria coronária. Por outro lado, o pedículo da ATI deixa de sofrer influência direta da insuflação pulmonar, especialmente em pacientes enfisematosos.

No presente caso houve uma complicação rara devido ao posicionamento da ATIE. Apesar de passada através de abertura no pericárdio, houve acotovelamento do pedículo arterial em conseqũência da maior distenção do tronco pulmonar. O simples reposicionamento do pendículo foi sufuciente para sanar o problema, sem a necessidade de circulação extracorpórea ou utilização de outros enxertos.

A finalidade do presente relato é destacar a complicação, que deverá ser considerada, já que, possivelmente, casos rotulados como espasmos da ATI, possam ser conseqüentes a acotovelamentos do pedículo arterial, de fácil resolução. 
LOURENÇÃO Jr., A.; IGLÉZIAS, J. C. R.; DALLAN, L. A.; PEREIRA, A. N. R.; OLIVEIRA, S. A.; JATENE, A. D. - Thoracic intern artery graft kinking in myocardial revascularization: a case report. Rev. Bras. Cir. Cardiovasc., 8 (3): 247-249, 1993.

ABSTRACT: The authors relate a successful surgical treatment of myocardial revascularization complicated by a thoracic intern artery graft kinking.

DESCRIPTORS: thoracic intern artery graft kinking; myocardial revascularization, surgery.

\section{REFERÊNCIAS BIBLIOGRÁFICAS}

1 FAVALORO, R. G. - Current status of coronary artery bypass graft (CABG) surgery. Semin. Thorac. Cardiovasc. Surg., 6: 67-71, 1994.

2 KIRKLIN, J. W. \& FISCH, C. - Guidelines and indications for coronary artery bypass graft surgery: a report of the American College of Cardiology/American Heart Association. Task Force on Assessment of Diagnostic and Therapeutic Cardiovascular Procedures (Subcommitee on Coronary Artery Bypass Graft Surgery). J. Am. Cardiol., 17: 543-589, 1991.
3 MILLS, N. L.; BRINGAZE III, W. L. - Preparation of the internal mammary artery graft. Which is the best method? J. Thorac. Cardiovasc. Surg., 98: 73-79, 1989.

4 PUIG, L. B.; FRANÇA NETO, L.; PONTES, R.; LOURENÇÃO JR., A.; RAMIREZ, J. A. F.; MACRUZ, R.; SILVA, E. E. R.; HUEB, W.; MYAZATO, A. N.; JATENE, F. B.; VERGINELLI, G.; PILEGGI, F.; JATENE, A. D. - Artéria mamária direita em posição retro-aórtica para revascularizaçāo direta do miocárdio. Arq. Bras. Cardiol., 42: 319-323, 1984. 